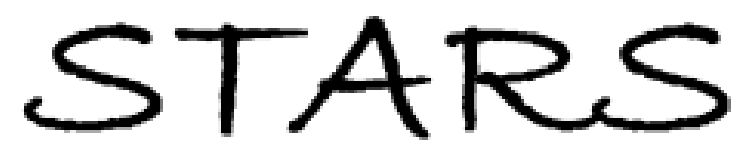

University of Central Florida

STARS

$1-1-2014$

\title{
Spatiotemporal cleaning of a femtosecond laser pulse through interaction with counterpropagating filaments in air
}

\author{
A. Jarnac \\ M. Durand \\ University of Central Florida \\ Y. Liu \\ B. Prade \\ M. Richardson \\ University of Central Florida
}

See next page for additional authors

Find similar works at: https://stars.library.ucf.edu/facultybib2010

University of Central Florida Libraries http://library.ucf.edu

This Article is brought to you for free and open access by the Faculty Bibliography at STARS. It has been accepted for inclusion in Faculty Bibliography 2010 s by an authorized administrator of STARS. For more information, please contactSTARS@ucf.edu.

\section{Recommended Citation}

Jarnac, A.; Durand, M.; Liu, Y.; Prade, B.; Richardson, M.; and Mysyrowicz, A., "Spatiotemporal cleaning of a femtosecond laser pulse through interaction with counterpropagating filaments in air" (2014). Faculty Bibliography 2010s. 5507.

https://stars.library.ucf.edu/facultybib2010/5507

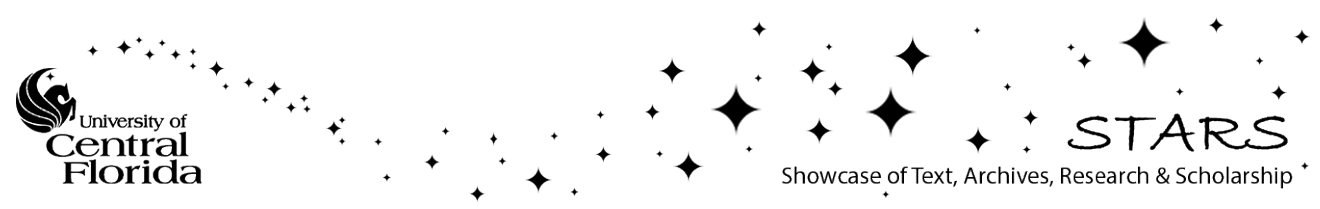


Authors

A. Jarnac, M. Durand, Y. Liu, B. Prade, M. Richardson, and A. Mysyrowicz 


\title{
Spatiotemporal cleaning of a femtosecond laser pulse through interaction with counterpropagating filaments in air
}

\author{
A. Jarnac, ${ }^{1}$ M. Durand, ${ }^{2}$ A. Houard,${ }^{1}$ Y. Liu, ${ }^{1}$ B. Prade, ${ }^{1}$ M. Richardson, ${ }^{2}$ and A. Mysyrowicz ${ }^{1, *}$ \\ ${ }^{1}$ Laboratoire d'Optique Appliquée, ENSTA Paristech, Ecole Polytechnique, CNRS, FR-91762 Palaiseau, France \\ ${ }^{2}$ Townes Laser Institute, CREOL, University of Central Florida, Orlando, Florida 32816, USA
}

(Received 10 June 2013; published 26 February 2014)

\begin{abstract}
We demonstrate spatiotemporal cleaning of a femtosecond laser pulse impinging on two counterpropagating filaments in air. The retroreflected signal essentially has a perfect beam profile. Prepulses present in the incident pulse are also efficiently removed. The performance of the "filament mirror" is explained by a plasma-mediated wave mixing process that can be viewed as a transient three-dimensional hologram.
\end{abstract}

DOI: 10.1103/PhysRevA.89.023844

PACS number(s): 42.65.Hw, 42.65.Jx, 52.38.Hb, 52.50.Jm

The propagation of an intense subpicosecond laser pulse in air, even with an energy of a few millijoules, is highly nonlinear. Two effects play a major role in inducing these strong optical nonlinearities during propagation. The first is the optical Kerr effect. By inducing a change in the air refractive index, it leads to beam self-focusing. Although weak at first, self-focusing is cumulative on propagation and becomes precipitous after some distance if the pulse initial peak power exceeds a critical value $p_{c r}=3.72 \lambda_{0}^{2} / 8 \pi n_{0} n_{2}$ which varies between 2.8 and $12 \mathrm{GW}$ at $800 \mathrm{~nm}$ in a normal atmosphere, depending upon pulse duration [1]. Here, $n_{0}$ is the linear refractive index, $n_{2}$ is the nonlinear index coefficient, and $\lambda_{0}$ is the laser wavelength. The second effect is high field ionization. Total beam collapse is prevented by the onset of ionization, a process requiring the simultaneous absorption of a large number of photons in air. The ensuing plasma is responsible for beam defocusing, an effect which clamps the pulse peak intensity to a value of $\sim 5 \times 10^{13} \mathrm{~W} / \mathrm{cm}^{2}[2,3]$. The dynamic competition between optical Kerr-induced self-focusing and plasma defocusing thus leads to the emergence of an intense contracted pulse propagating over distances exceeding its Rayleigh length. Due to its high intensity, the contracted pulse leaves, in its wake, a narrow string of underdense plasma. This highly nonlinear propagation regime is called filamentation [4].

In this paper, we examine, experimentally, the situation of three femtosecond laser pulses 1-3 crossing in air, two of which (1 and 2) are intense and consist of counterpropagating filamentary pulses. Pulse 3 is weaker and is noncollinear. In traditional nonlinear optics, a configuration of lower intensity counterpropagating beams form a phase conjugate mirror that retroreflects a noncollinear wave and inverts its spatial phase $[5,6]$. In a similar way, we detect a signal in the direction opposite to beam 3 . The counterpropagating filaments, thus, can be viewed as a transient mirror that retroreflects part of the incoming beam 3 . However, in contrast to the usual case of phase conjugation, the retroreflected beam (4) in the present case has a distortion-free profile, irrespective of the initial spatial phase of beam 3 . We also show that precursor light present in beam 3 is efficiently removed in the retroreflected

*Corresponding author: andre.mysyrowicz@ensta-paristech.fr pulse. To explain our results, we consider the interaction of the three beams in the high intensity limit.

This experiment illustrates the difference between traditional and femtosecond nonlinear optics. The high peak intensity of femtosecond laser pulses leads to specific effects, such as nonlinear propagation and plasma formation [4]. In addition, this experiment shows that two counterpropagating filaments provide a simple scheme to clean femtosecond laser pulses. Such pulses could find applications in ultrafast physics where femtosecond laser pulses with a high quality beam profile, good stability, and a high contrast are needed [7-9].

The principle of the experiment is shown in Fig. 1(a). Two ultrashort laser pulses 1 and 2 of equal peak power $>12 \mathrm{GW}$ undergo filamentation in air and then meet from opposite directions along $z$. The formation of a plasma bubble in the interaction zone is verified by the appearance of a bright spot superimposed on the blue filament luminescence tracks from ionized $\mathrm{N}_{2}$ molecules [10] as shown in Fig. 1(b). A weaker pulse 3 (beam diameter: $2 \mathrm{~mm}, P_{\max }=2 \mathrm{GW}$ ) is focused with an $f=150$-mm lens onto the bubble and is time coincident with the filament pulses. Due to the highly nonlinear ionization rate with the laser intensity, the size of the plasma shrinks to a small volume. Through luminescence imaging, we measured the shape of this bubble in the presence of the three pulses. It is an ellipsoid of revolution with an axis $11 \mu \mathrm{m}$ along $z$ and $30 \mu \mathrm{m}$ along $x$ and $y$. It induces two signal beams 4 and $4^{\prime}$, one counterpropagating with beam 3 , the other copropagating and amplifying it. All laser pulses 1-3 with central wavelengths at $800 \mathrm{~nm}$ and durations of $85 \mathrm{fs}$ are derived from the same Ti:sapphire laser. They are contained in the horizontal plane $(y, z)$ and are polarized along $x$.

Figure 2 shows an example of a beam profile before and after reflection on the filament mirror recorded with a CCD camera. Here, incident beam 3 propagates perpendicular to the filament paths $\left(\theta=90^{\circ}\right)$ and is intentionally of poor quality with a pronounced diffraction pattern and two holes in its fluence profile. By contrast, the retroreflected pulse 4 essentially has a single spatial mode profile [Fig. 2(b)]. We have verified that other strong initial beam distortions, such as those introduced by the masking part of the beam, are also removed. As shown in Fig. 3(a), the filament mirror reflectivity $R=W_{4} / W_{3}$ increases with decreasing angle $\theta$ between beams 1 and 3, where $W_{i}$ is the energy of pulse $i$. Figure 3(b) shows $R$ as a function of the incident filament pulse energy for a 


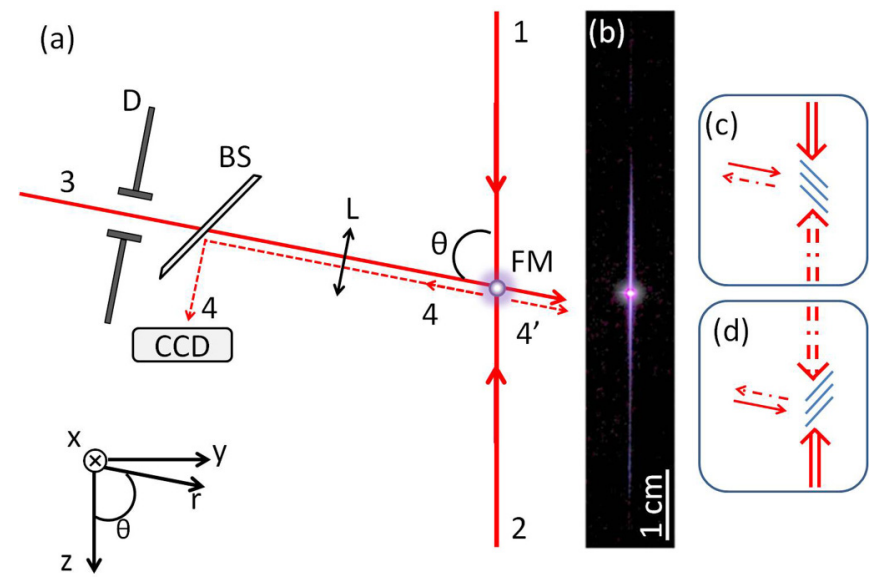

FIG. 1. (Color online) (a) Schematic setup: D: diaphragm; BS: beam splitter; L: lens; CCD: charge-coupled device camera; FM: filament mirror. (b) Photograph of the blue plasma luminescence emitted by the three overlapping beams; (c) and (d) schematics of the main diffraction process giving rise to signal beam 4 .

$\theta=90^{\circ}$ geometry. One notices a fast increase in the reflected signal 4 above $100 \mu \mathrm{J}$ when pulses 1 and 2 start ionizing air until a plateau is reached with a reflectivity of $10 \%-20 \%$. We have also measured an amplification of the transmitted energy of pulse 3 in the presence of the filament pulses. The amplified part $4^{\prime}$ is equal to the retroreflected signal 4 . It shows that laser energy is transferred from the filament pulses 1 and 2 to the signal pulses 4 and $4^{\prime}$.
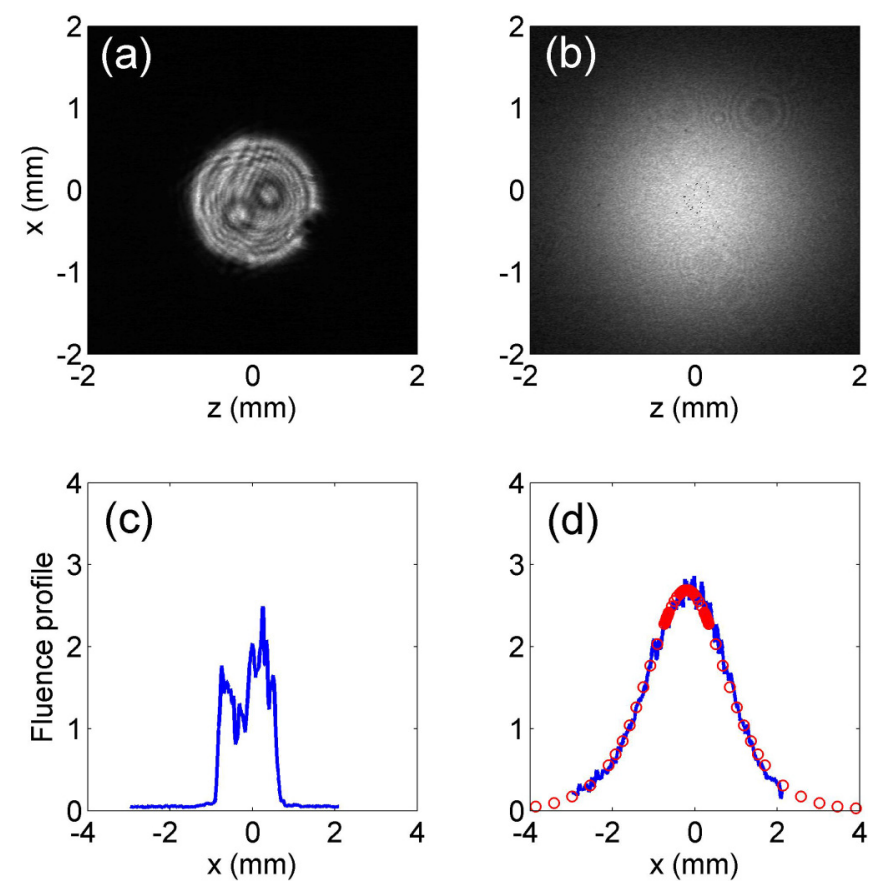

FIG. 2. (Color online) Fluence profile of a femtosecond pulse (a) before and (b) after reflection on the filament mirror as recorded in a single shot with a CCD camera. Scan of the fluence profile along $x$ at $z=0$ (c) before and (d) after reflection. The red open circles represent a fit of reflected beam 4 by a Townes mode profile function.
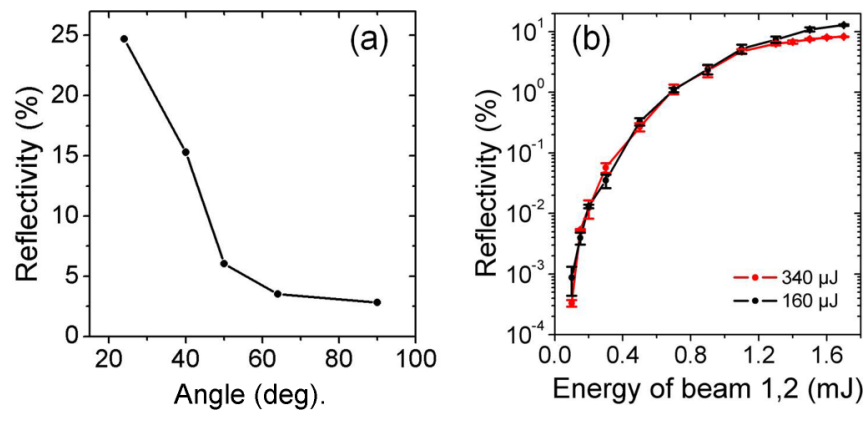

FIG. 3. (Color online) (a) Reflectivity $\left(R=W_{4} / W_{3}\right)$ of the filament mirror as a function of angle $\theta$ between pulses 1 and 3. Here $W_{1}=W_{2}=0.8 \mathrm{~mJ} ; W_{3}=170 \mu \mathrm{J}$. (b) Reflectivity as a function of pulses 1 and 2 energies. Pulse 3 has energies of 160 and $340 \mu \mathrm{J}$ and is incident at an angle of $90^{\circ}$. The three pulses are coincident in time.

To discuss these results, we examine the effect of a change in refractive index of air in the presence of the three waves. The superposition of the three waves 1-3 leads to a transient threedimensional (3D) plasma hologram that encodes the spatial phase profile of the three beams. The phase profile is dominated by the index variation from the plasma. It is of the form

$$
-\Delta n(t) \approx \int_{-\infty}^{t}\left[I\left(t^{\prime}\right)\right]^{N} d t^{\prime},
$$

where

$I\left(t^{\prime}\right)=\left|E_{1}\left(t^{\prime}-z / c\right)+E_{2}\left(t^{\prime}+z / c\right)+E_{3}\left(t^{\prime}-r / c\right)\right|^{2}$.

$N$ is the number of effective photons simultaneously absorbed to create the plasma, and $E_{i}$ is the complex electric field of wave $i$. For example, $E_{1}$ has the standard form

$$
E_{1}\left(t^{\prime}-\frac{z}{c}\right)=A_{1}\left(t^{\prime}-\frac{z}{c}\right) e^{i k_{1} z} e^{i \varphi_{1}(x, y)} e^{-i \omega t^{\prime}} .
$$

The holographic volume is simultaneously read by the three waves. We are concerned with the signal diffracted in the direction opposite to wave 3 .

It takes the general form

$$
E_{4}=F(t, x, y, z) e^{i \varphi_{1}} e^{i \varphi_{2}} e^{-i \varphi_{3}} e^{-i \vec{k}_{3} \cdot \vec{r}} e^{-i \omega t},
$$

where $F$ is a slowly varying amplitude that includes a number of terms that increases rapidly with $N$. It amounts to 40 terms in the case of $N=5$, which applies to a laser pulse at $800 \mathrm{~nm}$ [11]. Note that wave 4 carries the phase of the filament waves and the inverted phase of wave 3 . Pursuing the analogy with optical phase conjugation by degenerate four-wave mixing, we identify the main mechanism responsible for the generation of wave 4. In conventional degenerate four-wave mixing, a pure index grating diffracts part of the counterpropagating beams in direction $\vec{k}_{4}=-\vec{k}_{3}$. In our model, all incident beams contribute to wave 4 . However, the major contribution is due to beams 1 and 2: The plasma grating formed by filament pulse $1(2)$ and converging pulse 3 scatters part of the second filament pulse 2(1) at the Bragg angle into the direction opposite to the incident beam 3 [dashed-dotted lines in Figs. 1(c) and 1(d)]. Therefore, pulse 4 carries the properties of the intense pulses. Now a remarkable property of filamentation is spatial self-cleaning of the contracted beam profile [12-15], 


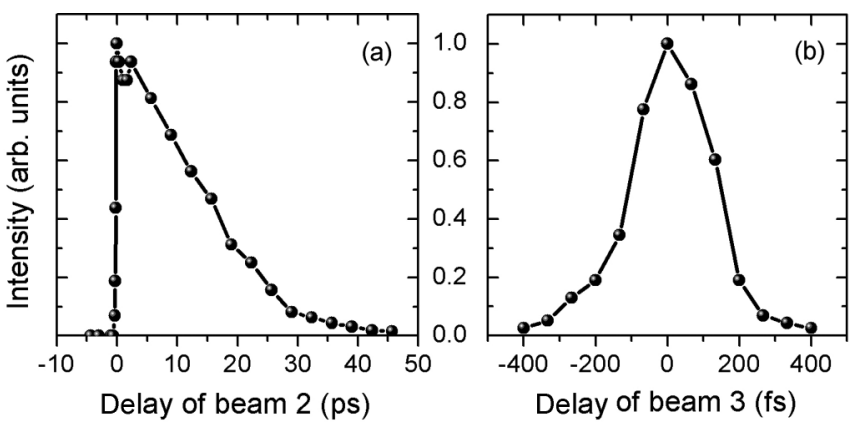

FIG. 4. (a) Signal of pulse 4 as a function of delay of filament pulse 2. Pulse 3 is incident at an angle of $90^{\circ}$. Pulses 1 and 3 are coincident in time. (b) The same but delaying pulse 3 with respect to pulses 1 and 2 .

which acquires a Townes mode profile [16]. We have verified that pulse 4, indeed, fits a Townes mode profile [see Fig. 2(d)]. An additional effect plays a role in removing phase distortions of beam 3, namely, the small size of the grating that acts as a filtering pinhole.

To confirm this interpretation, we have measured signal 4 as a function of delay of pulse 2 with respect to pulses 1 and 3 [see Fig. 4(a)]. The signal decays within 30 ps [17] because the plasma grating of the long lifetime in air can still diffract a delayed pulse 2 [18]. On the other hand, the signal vanishes within $100 \mathrm{fs}$ if pulse 3 is delayed with respect to the filament pulses [see Fig. 4(b)]. This is expected since the formation of the plasma grating requires the chronological coincidence of pulse 3 with at least one of the filamentary pulses.

The filament-based mirror also displays interesting temporal pulse cleaning properties. The steep initial increase in the reflectivity shown in Fig. 3(b) is responsible for the removal of laser pulse precursors. Indeed, the field interference between precursors present in the three pulses does not form a diffracting plasma grating due to their weaker intensities. On the other hand, the saturated reflectivity region above 1-mJ energy reduces shot-to-shot intensity fluctuations in pulse 4 .

We have verified experimentally the temporal cleaning property of the filament mirror, starting with a laser pulse with a marked temporal precursor, obtained by misaligning the compression stage of the laser. Figure 5 gives the time profile of the pulse (a) before and (b) after reflection by the filament mirror. The time profile and the temporal phase of the incident pulse 3 and reflected pulse 4 were obtained with a commercial frequency-resolved optical gating system apparatus (FROG). Figure 5(b) shows that the front pedestal
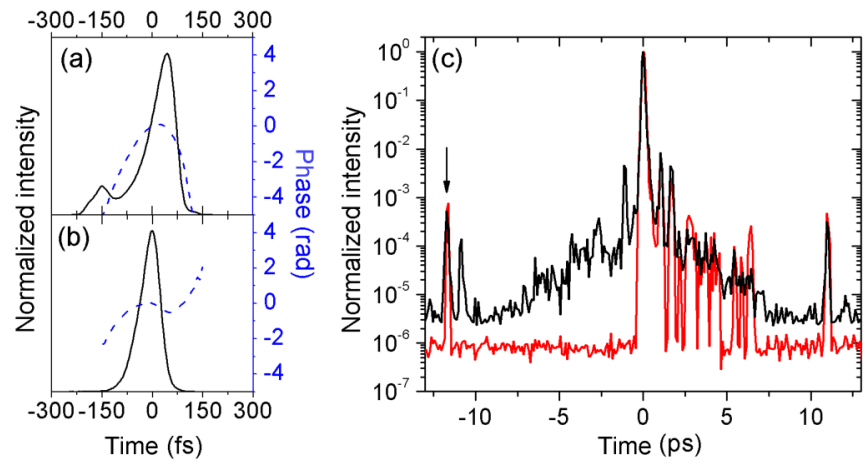

FIG. 5. (Color online) (a) Temporal profile of incident pulse 3 and (b) reflected pulse 4 measured with a FROG apparatus. (c) Contrast measurement performed with a $3 \omega$ cross correlator of laser pulse 3 before (black line) and after (red line) reflection by the filament mirror. The black arrow indicates an artifact of the correlator.

is totally removed after reflection on the filament mirror. The cleaning properties have also been measured over a larger time scale with a third-order cross correlator [19]. As can be seen in Fig. 5(c), pulse precursors are completely removed over the $10^{6}$ dynamic range of the cross correlator in the trace of the reflected pulse 4 .

Laser pulses with high contrast are desirable for studies of their interaction with intact samples. A prepulse, even of modest energy content, can preionize a sample and, thereby, can modify the conditions of interaction at the peak of the pulse [20]. Usual cleaning techniques are as follows: (i) the use of pinholes, which require strict alignment and are subject to damage [21], (ii) the cross-polarized wave method, which is fragile and expensive [22], or (iii) the plasma mirror, which requires a fresh sample after each shot [23]. A filament mirror is cheap since it uses air or another gas as a nonlinear material; it is not subject to damage and is relatively easy to align.

In conclusion, we have shown that it is possible to convert a short pulse of an arbitrary spatial profile into a single spatial mode pulse by reflecting it on a mirror formed by counterpropagating filaments. The mirror enhances the temporal contrast of the pulse by at least three orders of magnitude. Its action has been described in terms of a transient 3D plasma hologram. This hologram diffracts part of the filament's light in the direction opposite to that of a third incoming femtosecond laser pulse.

The authors thank G. Hamoniaux and P. Rousseau for technical help. This work was partially supported by DGA through a Ph.D. grant.
[1] J. K. Wahlstrand, Y-H. Cheng, and H. M. Milchberg, Phys. Rev. A 85, 043820 (2012).

[2] H. R. Lange, A. Chiron, J.-F. Ripoche, A. Mysyrowicz, P. Breger, and P. Agostini, Phys. Rev. Lett. 81, 1611 (1998).

[3] J. Kasparian, R. Sauerbrey, and S. L. Chin, Appl. Phys. B 71, 877 (2000).

[4] For a review, see A. Couairon and A. Mysyrowicz, Phys. Rep. 441, 47 (2007).
[5] Optical Phase Conjugation, edited by R. A. Fisher (Academic, New York, 1983).

[6] R. W. Boyd, Nonlinear Optics (Academic, Amsterdam, 2008), Chap. 7.

[7] T. Ceccotti, A. Lévy, H. Popescu, F. Réau, P. D’Oliveira, P. Monot, J. P. Geindre, E. Lefebvre, and P. Martin, Phys. Rev. Lett. 99, 185002 (2007).

[8] N. M. Naumova, J. A. Nees, I. V. Sokolov, B. Hou, and G. A. Mourou, Phys. Rev. Lett. 92, 063902 (2004). 
[9] J. A. Wheeler, A. Borot, S. Monchocé, H. Vincenti, A. Ricci, A. Malvache, R. Lopez-Martens, and F. Quéré, Nat. Photon. 6, 829 (2012).

[10] A. Talebpour, S. Petit, and S. L. Chin, Opt. Commun. 171, 285 (1999).

[11] S. L. Chin, in From Multiphoton to Tunnel Ionization, edited by S. H. Lin, A. A. Villaeys, and Y. Fujimura, Advances in Multiphoton Processes and Spectroscopy, Vol. 16 (World Scientific, Singapore, 2004), p. 249.

[12] B. Prade, M. Franco, A. Mysyrowicz, A. Couairon, H. Buersing, B. Eberle, M. Krenz, D. Seiffer, and O. Vasseur, Opt. Lett. 31, 2601 (2006).

[13] C. P. Hauri, W. Kornelis, F. W. Helbing, A. Heinrich, A. Couairon, A. Mysyrowicz, J. Biegert, and U. Keller, Appl. Phys. B 79, 673 (2004).

[14] X. Chen, Y. Leng, J. Liu, Y. Zhu, R. Li, and Z. Xu, Opt. Commun. 259, 331 (2006).

[15] F. Theberge, N. Akozbek, W. Liu, A. Becker, and S. L. Chin, Phys. Rev. Lett. 97, 023904 (2006).

[16] K. D. Moll, A. L. Gaeta, and G. Fibich, Phys. Rev. Lett. 90, 203902 (2003).

[17] M. Durand, A. Jarnac, Y. Liu, B. Prade, A. Houard, V. Tikhonchuk, and A. Mysyrowicz, Phys. Rev. E 86, 036405 (2012).
[18] Note that, when a short pulse 2 delay $\delta t$ is introduced, a fringe pattern becomes apparent in the spatial profile of pulse 4 with a fringe separation decreasing with delay. This pattern is due to the formation of a second displaced grating formed by interference of pulse 2 with the edge of pulse 3 . It disappears at delays $\delta t>150 \mathrm{fs}$.

[19] A. Jullien, O. Albert, F. Burgy, G. Hamoniaux, J.-P. Rousseau, J.-P. Chambaret, F. Augé-Rochereau, G. Chériaux, J. Etchepare, N. Minkovski, and S. M. Saltiel, Opt. Lett. 30, 920 (2005).

[20] M. Kaluza, J. Schreiber, M. I. K. Santala, G. D. Tsakiris, K. Eidmann, J. Meyer-ter-Vehn, and K. J. Witte, Phys. Rev. Lett. 93, 045003 (2004).

[21] P. M. Celliers, K. G. Estabrook, R. J. Wallace, J. E. Murray, L. B. Da Silva, B. J. MacGowan, B. M. Van Wonterghem, and K. R. Manes, Appl. Opt. 37, 2371 (1998).

[22] A. Ricci, A. Jullien, J.-P. Rousseau, Y. Liu, A. Houard, P. Ramirez, D. Papadopoulos, A. Pellegrina, P. Georges, F. Druon, N. Forget, and R. Lopez-Martens, Rev. Sci. Instrum. 84, 043106 (2013).

[23] C. Thaury, F. Quéré, J.-P. Geindre, A. Levy, T. Ceccotti, P. Monot, M. Bougeard, F. Réau, P. D’Oliveira, P. Audebert, R. Marjoribanks, and P. Martin, Nat. Phys. 3, 424 (2007) 